\title{
Metastatic Hepatocellular Carcinoma Presenting as Maxillary Sinus Lesion
}

\author{
KG Somashekara ${ }^{1}$, Lakshmi Shantharam², GM Bilkhis Mahjabeen ${ }^{3}$
}

\begin{abstract}
Metastasis of malignant tumors to the sinonasal area is an infrequent occurrence and usually indicates the late stage of the primary disease. We report a rare case of previously undiagnosed metastatic hepatocellular carcinoma presenting solely as a maxillary sinus lesion. We have also reviewed literature on hepatocellular carcinoma metastasizing to the sinonasal area and discussed the pathogenesis of the possible routes of metastatic spread.

Keywords: Hepatocellular carcinoma, Maxillary sinus, Metastasis.

International Journal of Head and Neck Surgery (2020): 10.5005/jp-journals-10001-1345
\end{abstract}

\section{INTRODUCTION}

Metastatic lesions involving the nose and paranasal sinuses (PNS) are rare. Hepatocellular carcinoma metastasizing to the nose and paranasal sinuses is even rarer. We report a case of metastatic hepatocellular disease which presented as a lesion in the maxillary sinus before the primary disease was diagnosed. Clinicians should always be aware of such unusual presentations as such cases can deteriorate rapidly and they are almost always associated with a dismal prognosis. ${ }^{1}$

\section{Case Report}

A 77-year-old man presented to the ENT OPD with complaints of pain and swelling over the right cheek, watering and burning sensation in the right eye, drooping of the right eyelid, and diplopia for 2 months (Fig. 1). There was a history of unilateral nasal obstruction and occasional epistaxis. The patient was a known diabetic and was on treatment for 4 years. There was also a history of tobacco snuff usage since adolescence. On examination, there was a diffuse, firm, tender swelling over the right cheek. On diagnostic nasal endoscopy, there was a bulge in the lateral

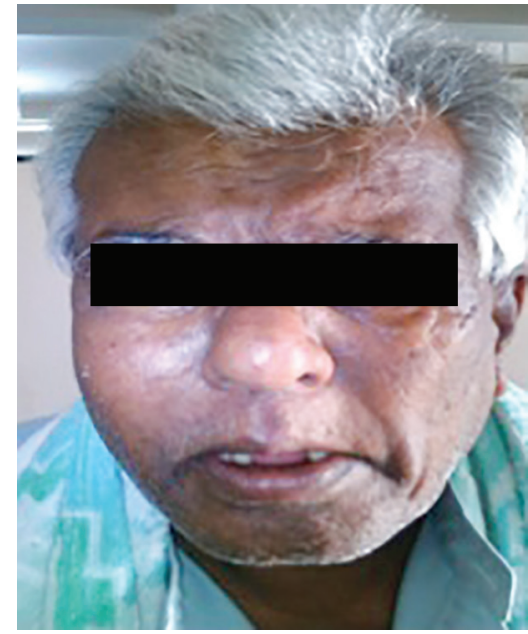

Fig. 1: Patient presented with right cheek swelling and right eye ptosis

\begin{abstract}
${ }^{1-3}$ Department of ENT, Kempegowda Institute of Medical Sciences, Bengaluru, Karnataka, India

Corresponding Author: KG Somashekara, Department of ENT, Kempegowda Institute of Medical Sciences, Bengaluru, Karnataka, India, Phone: +91 9844024597, e-mail: kgsomashekara@gmail.com

How to cite this article: Somashekara KG, Shantharam L, Mahjabeen GMB. Metastatic Hepatocellular Carcinoma Presenting as Maxillary Sinus Lesion. Int J Head Neck Surg 2020;11(1):19-21.

Source of support: Nil

Conflict of interest: None
\end{abstract}

wall of the right nasal cavity at the level of middle meatus. On ophthalmological examination, there was a restriction of eyeball movements inferiorly. Fundoscopy was normal. Routine blood and urine investigations showed raised erythrocyte sedimentation rate (ESR) and deranged lipid profile. Liver function tests were normal. CT PNS showed a lesion in the right maxillary sinus with destruction of its walls. Also the fat plane between the inferior rectus and lesion was lost suggesting infiltration (Figs 2 and 3).

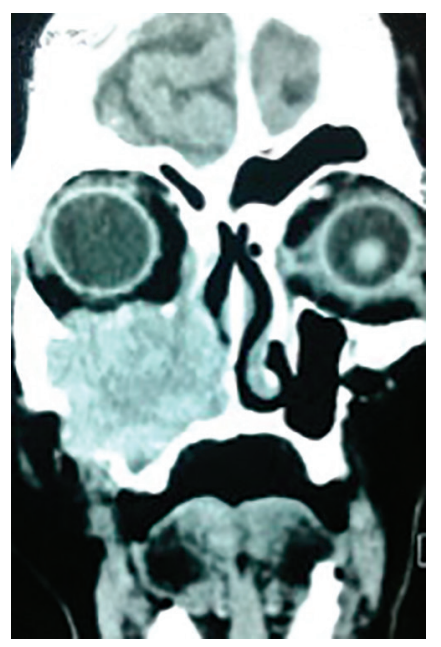

Fig. 2: CT PNS showed a lesion in the right maxillary sinus with destruction of its walls. Fat plane between the inferior rectus and the lesion was lost suggesting infiltration

(-) The Author(s). 2020 Open Access This article is distributed under the terms of the Creative Commons Attribution 4.0 International License (https://creativecommons. org/licenses/by-nc/4.0/), which permits unrestricted use, distribution, and non-commercial reproduction in any medium, provided you give appropriate credit to the original author(s) and the source, provide a link to the Creative Commons license, and indicate if changes were made. The Creative Commons Public Domain Dedication waiver (http://creativecommons.org/publicdomain/zero/1.0/) applies to the data made available in this article, unless otherwise stated. 


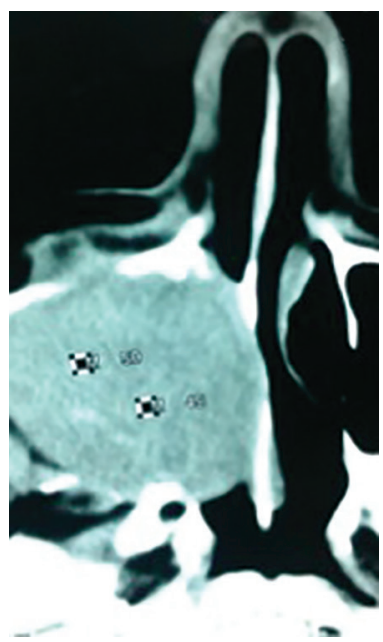

Fig. 3: CT PNS showing maxillary sinus and tumor destroying the walls. Fat plane between the inferior rectus and the lesion was lost suggesting infiltration

The diagnostic nasal endoscopy was repeated and a nick was made over the lateral wall bulge which revealed a protruding mass. Biopsy was done following which there was torrential bleeding. It was controlled with anterior nasal packing. Histopathological analysis was suggestive of metastatic hepatocellular carcinoma. On further evaluation, multifocal hepatic lesions suggestive of hepatocellular carcinoma with secondaries were discovered on abdomen CT. It also showed bilateral adrenal nodular metastatic deposits, a thin wall nonenhancing uniloculated cyst in the tail of pancreas, and a lytic lesion involving the left ischium and L1 vertebra. PET CT scan revealed few scattered tiny nodular lesions in bilateral lung parenchyma. A panel of immunohistochemical studies on the maxillary sinus lesion showed neoplastic cells staining positive for Hep Par 1 (Fig. 4) and cytokeratin. This confirmed the diagnosis of metastatic hepatocellular disease.

\section{Discussion}

Malignant lesions of the sinonasal region constitute less than $1 \%$ of all malignancies, majority of these being primary in origin with metastatic lesions being relatively rare. ${ }^{2}$ The most common primary sources to metastasize to the sinonasal area in decreasing order are kidneys (49\%), lungs (12\%), urogenital area (12\%), breast (9\%), and gastrointestinal tract (6\%). ${ }^{3,4}$

Hepatocellular carcinoma, which is among the most common malignancies around the world, has a high potential for metastasis. ${ }^{5}$ At the moment of diagnosis, about $50 \%$ of the patients have distant metastasis. ${ }^{6}$ But metastasis of hepatocellular carcinoma to the head and neck regions is uncommon. ${ }^{7}$ The most frequent metastatic sites of hepatocellular carcinoma are lungs (18.1-49.2\%), lymph nodes (26.5-41.7\%), adrenal glands (8.4-15.4\%), and bones (4.2-16.3\%). ${ }^{8}$ Very rarely, cases of hepatocellular carcinoma metastasizing to mandible, maxilla, orbital cavity, oral cavity, larynx, pituitary gland, nasal cavity, and paranasal sinuses have been reported. ${ }^{9}$

Therefore, hepatocellular carcinoma metastasizing to the sinonasal area is indeed a very rare occurrence.

To our knowledge, only 25 cases of hepatocellular carcinoma metastasizing to the sinonasal area are reported in the literature, in which 10 cases had metastasis to maxillary sinus. ${ }^{7-16}$

Most of the patients with hepatocellular carcinoma metastasizing to the sinonasal region were men and in the age

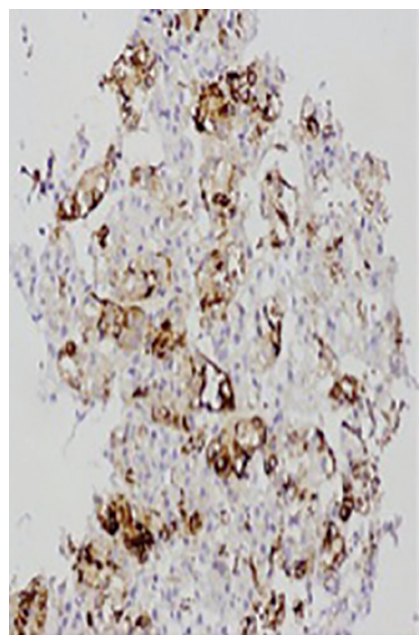

Fig. 4: Immunohistochemical study of the biopsy of the maxillary sinus lesion showing neoplastic cells positive for Hep Par 1 antibody

group of 50-70 years. ${ }^{7}$ According to Okada, this gender and age predilection is so because the primary hepatocellular carcinoma occurs mostly in men aged $45-60$ years, and it takes several years to metastasize to the sinonasal area. ${ }^{10}$

Epistaxis was the presenting symptom in majority of the patients, more so if the metastasis was in the maxillary sinus., ${ }^{711}$ Headache, diplopia, and visual impairment were more common with tumors in the sphenoid sinus. Our case, on the contrary, presented with cheek pain and eye symptoms such as ptosis, diplopia, and watering of the eye.

The pathogenesis of the metastatic spread of hepatocellular carcinoma could be through both haematogenous and lymphatic routes. In cases where metastasis is found in the lungs, the spread could be via the conventional route, i.e., the caval venous system thence to the pulmonary circulation and then from the heart to the sinuses via the arteries of the head and neck region. ${ }^{3,4}$ In cases where metastasis was not found in the lungs, Nahum and Bailey proposed an unusual retrograde pattern of tumor spread. ${ }^{4}$ A retrograde flow occurs in the prevertebral and vertebral venous system when there is raised intrathoracic or intra-abdominal pressures as there are no valves. Such a retrograde flow could reach as far as the pterygoid plexus, cavernous sinus, and superior portion of pharyngeal plexus and hence reach the paranasal sinuses.

Metastasis to the sinonasal region is associated with advanced disease and poor clinical outcome. ${ }^{12}$ The mean survival of such patients ranges from 4 weeks to 26 months. ${ }^{11,12}$ Treatment modalities for such extensive metastasis include palliative radiotherapy, systemic chemotherapy, and symptomatic palliation such as transcatheter embolization to control nasal bleeding or surgical excision and electrocautery of a bulky tumor., ${ }^{1,11,12}$

As is seen in this case, the symptoms and signs of the sinonasal metastasis may be the initial manifestation of advanced malignancies. Since the prognosis is poor and outcome is fatal, metastatic tumors should always be included in the differential diagnosis of rapidly growing sinonasal lesions. ${ }^{1}$

\section{References}

1. Liu CY, Chang LC, et al. Metastatic hepatocellular carcinoma to the nasal cavity: A case report and review of literature. J Cancer Sci Ther 2011;3:081-083. DOI: 10.4172/1948-5956.1000064.

2. Carrau RL, Myers EN. Neoplasms of the nose and paranasal sinuses. In: Bailey BJ, Calhoun KH, et al. ed: Head and Neck 
Surgery - Otolaryngology, 3rd ed. Philadelphia: Lippincott Williams \& Wilkins; 2001. pp. 1247-1264.

3. Bernstein JM, Montgomery WM, et al. Metastatic tumours of the maxilla, nose and paranasal sinuses. Laryngoscope 1966;76:621-650. DOI: 10.1288/00005537-196604000-00003.

4. Nahum AM, Bailey BJ. Malignant tumours metastatic to the paranasal sinuses: Case report and review of the literature. Laryngoscope 1963;73:942-953. DOI: 10.1288/00005537-196307000-00009.

5. Lin CD, Cheng KS, et al. Metastatic hepatocellular carcinoma in the nasal septum: report of a case. J Formos Med Assoc 2002;101(10):715-718.

6. Kim HS, Shin JW, et al. Metastasis of hepatocellular carcinoma to the small bowel manifested by intussusception. World J Gastroenterol 2006;12(12):1969-1971. DOI: 10.3748/wjg.v12.i12.1969.

7. Huang $\mathrm{H}-\mathrm{H}$, Chang $\mathrm{P}-\mathrm{H}$, et al. Sinonasal metastatic hepatocellular carcinoma. Am J Otolaryngol 2007;28:238-241. DOI: 10.1016/ j.amjoto.2006.08.012.

8. Kolarevic D, Tomasevic Z, et al. Metastasis of hepatocellular carcinoma presented as a tumour of the maxillary sinus and retrobulbar tumour. Vojnosanit Pregl 2011;68(4):359-362. DOI: 10.2298/VSP1104359K.

9. Aung $\mathrm{TH}, \mathrm{Po} \mathrm{YC}$, et al. Hepatocellular carcinoma with metastasis to the skull base, pituitary gland, sphenoid sinus and cavernous sinus. Hong komg Med J 2002 Feb;8(1):48-51.
10. Okada $\mathrm{H}$, Kamino $\mathrm{Y}$, et al. Metastatic hepatocellular carcinoma of maxillary sinus: A rare autopsy case without lung metastasis and a review. Int J. Oral Maxillofacial Surg 2003;32:97-100. DOI: 10.1054/ ijom.2002.0242.

11. Hwang CS, Kim YS, et al. A case of extrahepatic metastasis of Hepatocellular carcinoma in nasal septum managed with endoscopic resection. J Rhinol 2012;19(1):74-76.

12. Matsuda $H$, Tanigaki $Y$, et al. A case of metastatic hepatocellular carcinoma in the nasal cavity. Eur Arch Otorhinolaryngol 2005;263:305-307. DOI: 10.1007/s00405-005-1000-8.

13. Tandon S, Nair A, et al. Hepatocellular carcinoma presenting as an isolated sphenoid sinus lesion. Ear Nose throat J 2012 Jan;91(1):E10-E13. DOI: 10.1177/014556131209100116.

14. Chang CW, Wang TE, et al. Unusual presentation of metastatic hepatocellular carcinoma in the nasal septum. A case report and review of the literature. Med Oncol 2008;25:264-268. DOI: 10.1007/ s12032-007-9028-9.

15. Kourelis $\mathrm{K}$, Chen $\mathrm{T}$, et al. Metastatic hepatoma of maxillary sinus with atypical presentation. ANZ J Surg 2012;82:555-557.

16. Satake N, Yoshida S, et al. Adenoid cystic carcinoma of maxillary sinus with metastatic hepatocellular carcinoma. APMIS 2005;113:450-455. DOI: 10.1111/j.1600-0463.2005.apm_137.x. 